\title{
BESTÄTIGTE FÄLLE VON GEBÄRMUTTERHALSKREBS IN BRASILIEN ZWISCHEN 2010 UND 2014
}

\section{ORIGINALER ARTIKEL}

FARIAS, Rafaela Santos ${ }^{1}$, FACCO, Lucas², FECURY, Amanda Alves ${ }^{3}$, ARAÚJO, Maria Helena Mendonça de ${ }^{4}$, OLIVEIRA, Euzébio de ${ }^{5}$, DENDASCK, Carla Viana ${ }^{6}$, SOUZA, Keulle Oliveira da ${ }^{7}$, DIAS, Claudio Alberto Gellis de Mattos ${ }^{8}$

FARIAS, Rafaela Santos. Et al. Bestätigte Fälle von Gebärmutterhalskrebs in Brasilien zwischen 2010 und 2014. Revista Científica Multidisciplinar Núcleo do Conhecimento. Jahrgang 05, Ed. 11, Vol. 25, S. 93-104. November 2020. ISSN: 2448-0959, Zugriffsverbindung: https://www.nucleodoconhecimento.com.br/gesundheit/gebaermutterhalskrebs, DOI: 10.32749/nucleodoconhecimento.com.br/gesundheit/gebaermutterhalskrebs

\section{ZUSAMMENFASSUNG}

Der Pap-Abstrich (PCCU) ist eine gynäkologische Untersuchung, die bei Frauen durchgeführt wird, um Schäden am Gebärmutterhals zu erkennen. Veränderungen können als Gewebestörung beobachtet werden, die es ausmacht. Ziel dieser Arbeit ist es, bestätigte Fälle von Gebärmutterhalskrebs in Brasilien zwischen 2010 und 2014 aufzuzeigen. Die Forschung wurde mit Daten aus DATASUS

\footnotetext{
${ }^{1}$ Bergbautechnik, als Ergebnis des Bundesinstituts für Amapá (IFAP).

2 Student des Medizinkurses der Föderalen Universität Amapá (UNIFAP).

${ }^{3}$ Biomedizin, PhD in Tropenkrankheiten, Professor und Forscher des Medizinischen Studiengangs der Föderalen Universität Amapá (UNIFAP).

${ }^{4}$ Arzt, Professor und Forscher des Medizinischen Studiengangs der Föderalen Universität Amapá (UNIFAP).

${ }^{5}$ Biologe, PhD in Topische Krankheiten, Professor und Forscher des Leibeserziehungskurses der Föderalen Universität Pará (UFPA).

${ }^{6}$ Theologe, PhD in Psychoanalyse, Forscher am Center for Research and Advanced Studies - CEPA.

${ }^{7}$ Soziologe, Master-Student in Anthropischen Studien im Amazonas, Mitglied der Forschungsgruppe "Labor für Bildung, Umwelt und Gesundheit" (LEMAS/UFPA).

${ }^{8}$ Biologe, PhD in Theorie- und Verhaltensforschung, Professor und Forscher des Graduiertenprogramms für berufliche und technologische Bildung (PROFEPT), Bundesinstitut für Amapá (IFAP).
}

RC: 67821

Disponível em: 
(http://datasus.saude.gov.br) durchgeführt. Wenn es eine gute Leistung gibt, um eine Krankheit zu verhindern, die als schwerwiegend angesehen wird, ist das Ergebnis zufriedenstellend und wirksam. Durch die Ergebnisse, die durch die Analyse der Daten zwischen den Jahren 2010 bzw. 2014 erzielt wurden, wird der Schluss gezogen, dass im Laufe der Jahre die Suche nach dem Test allmählich zugenommen hat, aber einige Aspekte blieben bestehen, wie die Scham, den pccTest durchzuführen, Angst, Schmerzen, unter anderem. Die Ergebnisse zeigten jedoch eine hohe Testrate in der südöstlichen Region und eine niedrigere Rate im Norden, mit geringer Verbreitung und geringem Bewusstsein für Frauen in der nördlichen Region. Daher ist die Verbreitung, das Bewusstsein und die Durchführung der zytopathologischen Untersuchung sehr wichtig für die Prävention von Gebärmutterhalskrebs, der heute die Hauptursache für die Sterblichkeit bei brasilianischen Frauen ist.

Schlüsselwörter: Epidemiologie, Gebärmutterhalskrebs, PCCU, Zytopathologie.

\section{EINFÜHRUNG}

Es werden Vorsorgeuntersuchungen durchgeführt, um mögliche Krankheiten zu identifizieren und sie in ihren Anfängen zu behandeln (BRASIL, 2013). Der PapAbstrich (PCCU) ist eine gynäkologische Untersuchung bei Frauen, um Schäden am Gebärmutterhals zu erkennen, die gesundheitsschädlich werden können (TEIXEIRA et al., 2015). Dieser Test wird verwendet, um frühe Veränderungen im Gebärmutterhals zu entdecken. Es hilft bei der Frühdiagnose von Weiblichem Krebs und kann in seinem Ausgangszustand behandelt werden, wodurch eine Erhöhung der Sterblichkeitsrate bei Frauen vermieden wird (ARA-JO et al., 2016). Diese Untersuchung sollte von Frauen durchgeführt werden, die ein sexuell aktives Leben führen oder älter als 18 Jahre sind. Es beruht auf der Sammlung und Analyse von Gebärmutterhalsproben, um Krebs zu identifizieren (BARBER et al., 2009).

$\mathrm{RC}: 67821$

Disponível em: 
Veränderungen können als Gewebestörung beobachtet werden, die den Gebärmutterhals ausmacht. Diese Störung kann in Grade unterteilt werden. Grad 1 (I) tritt auf, wenn eine leichte Störung der Zellen auftritt, die kleine Teile des Gewebes beeinträchtigt. Wenn diese zelluläre Störung bis zu drei Viertel des Gewebes erreicht, wird sie in Grad 2 (II) berücksichtigt. In Grad 3 (III) wird beobachtet, dass die Anordnung der Zellen völlig ungeordnet ist, wodurch der sogenannte Gebärmutterhalskrebs identifiziert wird (MENETRIER et al., 2016).

Die Zahl der PCCU-Prüfungen, die 2010 in Brasilien durchgeführt wurden, betrug etwa 10 Millionen Prüfungen pro Jahr. Diese Menge an Pap-Abstrich-Scans variierte zwischen brasilianischen Regionen. In der nördlichen Region wurden 603.738 (sechshundertdreitausend siebenhundertachtunddreißig) Prüfungen durchgeführt. Die nordöstliche Region führte 2.616.344 (zwei Millionen sechshundertsechzehntausend dreihundertvierundvierzig) Prüfungen durch. Im Mittleren Westen wurden $745.875 \quad$ (siebenhundertfünfzigtausend achthundertfünfundsiebzig) Prüfungen durchgeführt. Im Südosten wurden 4.703.400 (vier Millionen siebenhundertdreihundert vierhundert) Menschen untersucht. In der $\begin{array}{lllll}\text { südlichen } & \text { Region } & \text { wurden } & 1.606 .119 & \text { (eine }\end{array}$ sechshundertsechstausendhundert1zehn) Prüfungen (BORTOLON et al., 2012).

Die Anzahl der PCCU-Prüfungen, die 2010 in der nördlichen Region Brasiliens durchgeführt wurden, variiert je nach Bundesland. In Amapa wurden 19.657 (neunzehntausend sechshundertfünfzig) Prüfungen durchgeführt. In Acre wurden 53.754 (dreiundfünfzigtausend siebenhundertvierundfünfzig) Prüfungen durchgeführt. $\begin{array}{llll}\text { In amazonas } & 134.268 & \text { (Einhundertvierunddreißigtausend }\end{array}$ zweihundertachtundsechzig) Prüfungen durchgeführt. Im Bundesstaat Para, 191,298 (Einhunderteinneunzigtausend zweihundertachtundneunzig) Prüfungen. In Rondénia wurden 92.085 (zweiundneunzigtausend und zweiundachtzig) Prüfungen durchgeführt. Im Bundesstaat Roraima wurden 28.614 (achtundzwanzigtausend sechshundertvierzehnjahre) Prüfungen durchgeführt. In Tocantins wurden 84.062

RC: 67821

Disponível em:

https://www.nucleodoconhecimento.com.br/gesundheit/gebaermutterhalskrebs 
(vierundachtzigtausend und zweiundsechzig) Tests durchgeführt (BORTOLON et al., 2012).

\section{TOY}

Zeigen Sie bestätigte Fälle von Gebärmutterhalskrebs in Brasilien zwischen 2010 und 2014.

\section{SYSTEM}

Die Forschung wurde mit Daten aus DATASUS (http://datasus.saude.gov.br) nach folgenden Schritten durchgeführt: Zuerst wurde die Registerkarte "Zugang zu Informationen" ausgewählt, gefolgt von der Option "Gesundheitsinformationen (TABNET)" kurz nach der Unteroption "Epidemiologisch und Morbidität" nach dem Zugriff auf die Optionsgruppe "Gebärmutterhals- und Brustkrebs (SISCOLO/SISMAMA). Als nächstes wurde die Ikone "Gebärmutterhals- und Brustkrebs - seit 2000" ausgewählt. Die Registerkarte "Siscolo 4.00 oder höher" wurde geöffnet und die Option "Cervico-vaginale zytopathologische Untersuchung und Mikroflora-Verfahren 12.011.01-0" ausgewählt. Dann klickte auf "Brasilien/Regionen". Für die Datenerfassung wurde im Zeilenfeld die Option "Jahr der Kompetenz" ausgewählt. Im Inhaltsfeld wurde die Option "Anzahl der Prüfungen" ausgewählt. Im Spaltenfeld waren die Optionen "Nicht aktiv"; "Jahr der Kompetenz"; Jahr der Kompetenz"; "Reg.residéncia"; "Schule"; "Farbe/Rasse"; "Innerhalb normaler Grenzen"; "Interv sammelt"; "Interv-Ergebnis"; "Cytopatol.Vorherige"; und "Elt Time.Verhindern." Für alle Optionen wurden Daten von 2010 bis 2014 erhoben. Die Option "Monat/Jahr der Kompetenz" wurde auch im Spaltenfeld im Spaltenfeld und im Inhaltsfeld die Option "Anzahl der Prüfungen" für die gleichen Jahre ausgewählt. Die Daten wurden in der Excel-Anwendung, einer Komponente der Microsoft Corporation Office-Suite, kompiliert. Die bibliographische Forschung wurde in wissenschaftlichen Artikeln durchgeführt, mit hilfe für die Suchcomputer des Computerlabors des Bundesinstituts für Bildung, Wissenschaft und Technologie von

RC: 67821

Disponível em:

https://www.nucleodoconhecimento.com.br/gesundheit/gebaermutterhalskrebs 
Amap, Cémpus Macapa, mit Sitz: Rodovia BR 210 KM 3, s/n - Bairro Brasil Novo, CEP: 68.909-398, Macapa, Amap, Brasilien.

\section{ERGEBNISSE}

Abbildung 1 zeigt den Prozentsatz der Pap-Abstrich-Scans (PCCU), die zwischen 2010 und 2014 in Brasilien durchgeführt wurden. Zwischen 2010 und 2013 blieb die Zahl der Tests praktisch stabil. Im Jahr 2014 zeigen die Daten, dass die Leistung der Prüfungen im Land stark abnahm.

Abbildung 1 zeigt den Prozentsatz der Pap-Abstrich-Scans (PCCU), die zwischen 2010 und 2014 in Brasilien durchgeführt wurden.

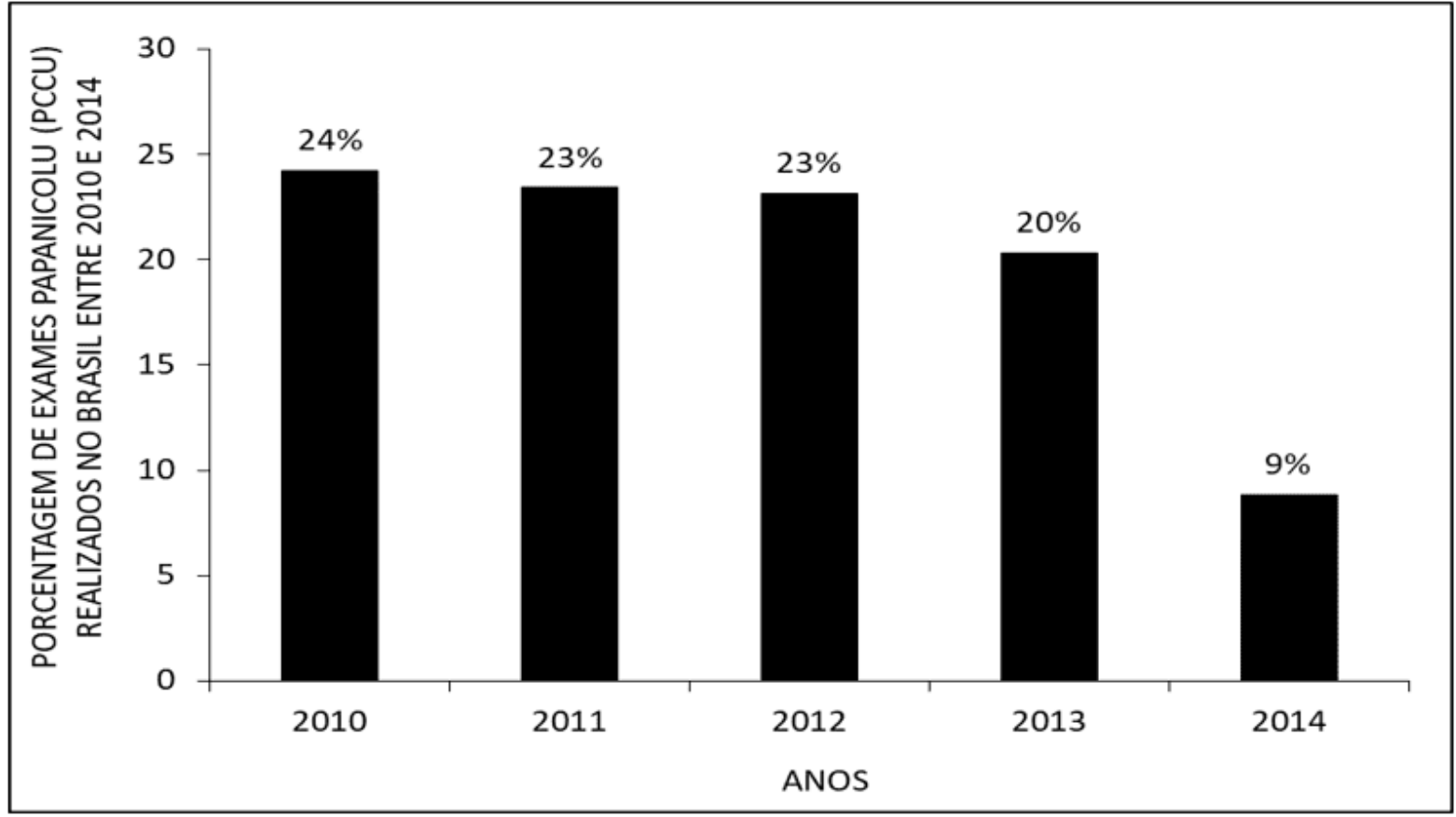

Abbildung 2 zeigt den prozentualen Anteil der Pap-Abstrich-Scans, die zwischen 2010 und 2014 in Brasilien durchgeführt wurden, nach Regionen des Landes. Zeigt eine höhere Rate von Tests in der südöstlichen Region und eine geringere Anzahl von Tests in den Regionen Nordosten, Süden, Mittlerer Westen und Norden.

RC: 67821

Disponível em: 
Abbildung 2 zeigt den prozentualen Anteil der Pap-Abstrich-Scans (PCCU), die in Brasilien zwischen 2010 und 2014 nach Regionen des Landes durchgeführt wurden.

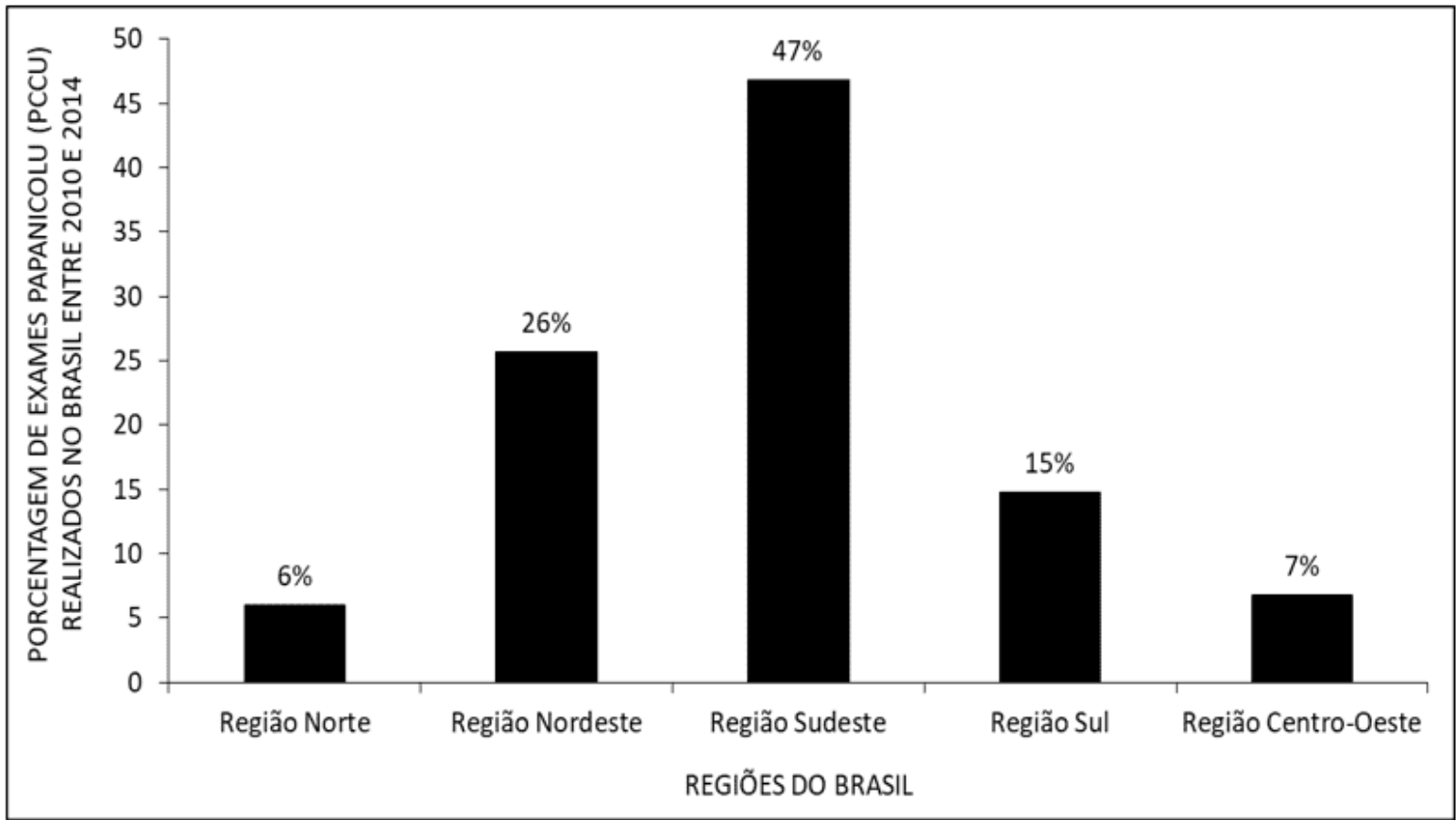

Abbildung 3 zeigt den prozentualen Anteil der Pap-Abstrich-Scans (PCCU), die in Brasilien zwischen 2010 und 2014 durchgeführt wurden, je nach Altersgruppe. Die Daten zeigen, dass die meisten Tests bei Frauen im Alter von 30 bis 39 Jahren durchgeführt werden. Die kleinste ist in der Altersgruppe der Frauen bis 19 Jahre.

RC: 67821

Disponível em: 
Abbildung 3 zeigt den Prozentsatz der Pap-Abstrich-Scans (PCCU), die zwischen 2010 und 2014 in Brasilien durchgeführt wurden. je nach Altersgruppe.

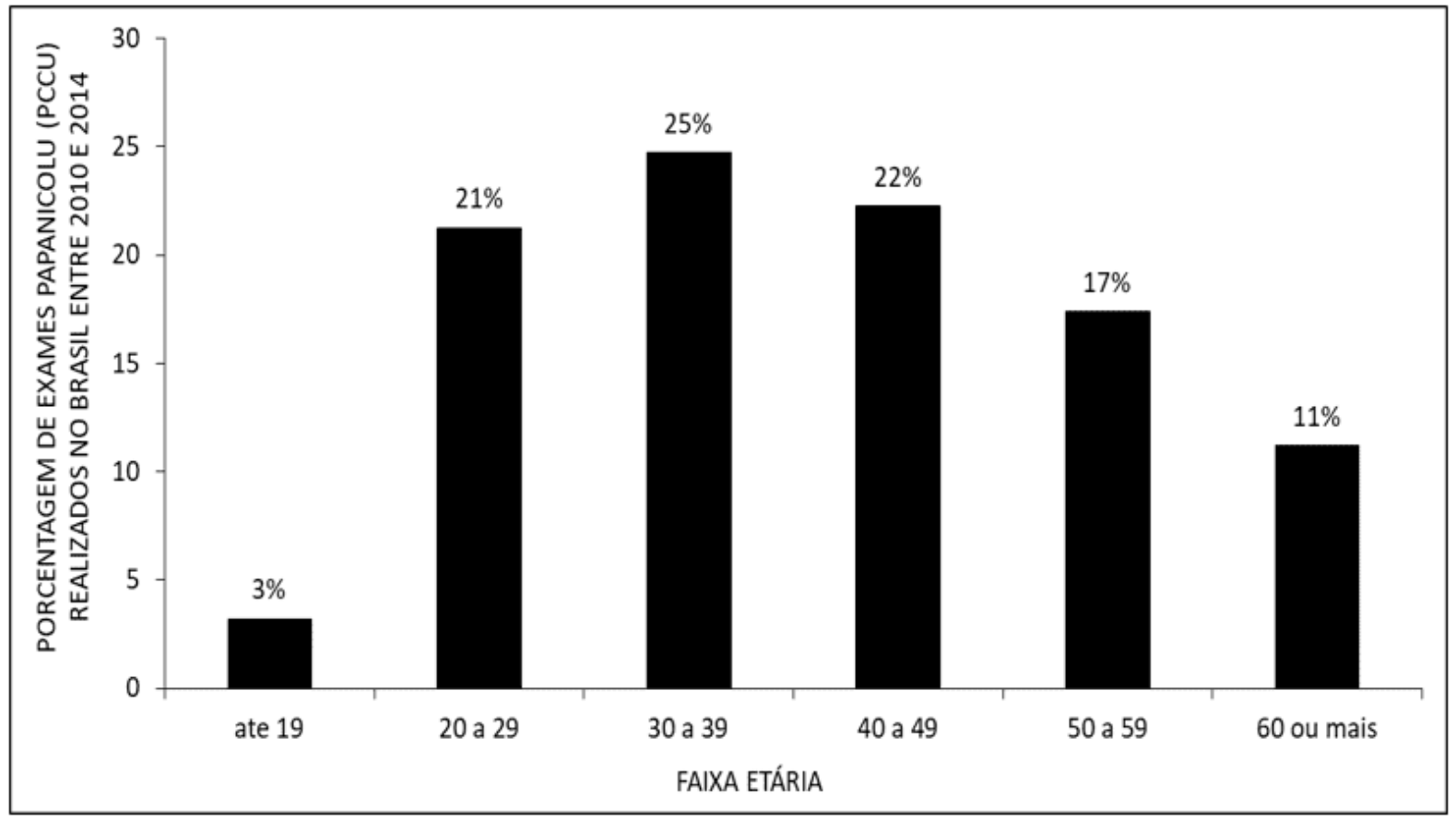

Abbildung 4 zeigt den Prozentualen Anteil der Pap-Abstrich-Scans (PCCU), die in Brasilien zwischen 2010 und 2014 durch Schulbildung durchgeführt wurden. Zeigt, dass die meisten Frauen, die die Prüfung ablegen, eine unvollständige Grundschule haben. Während der kleinste Betrag die Hochschulbildung abgeschlossen hat.

RC: 67821

Disponível em: 
Abbildung 4 zeigt den Prozentualen Anteil der Pap-Abstrichtests (PCCU), die in Brasilien zwischen 2010 und 2014 durch Schulbildung durchgeführt wurden.

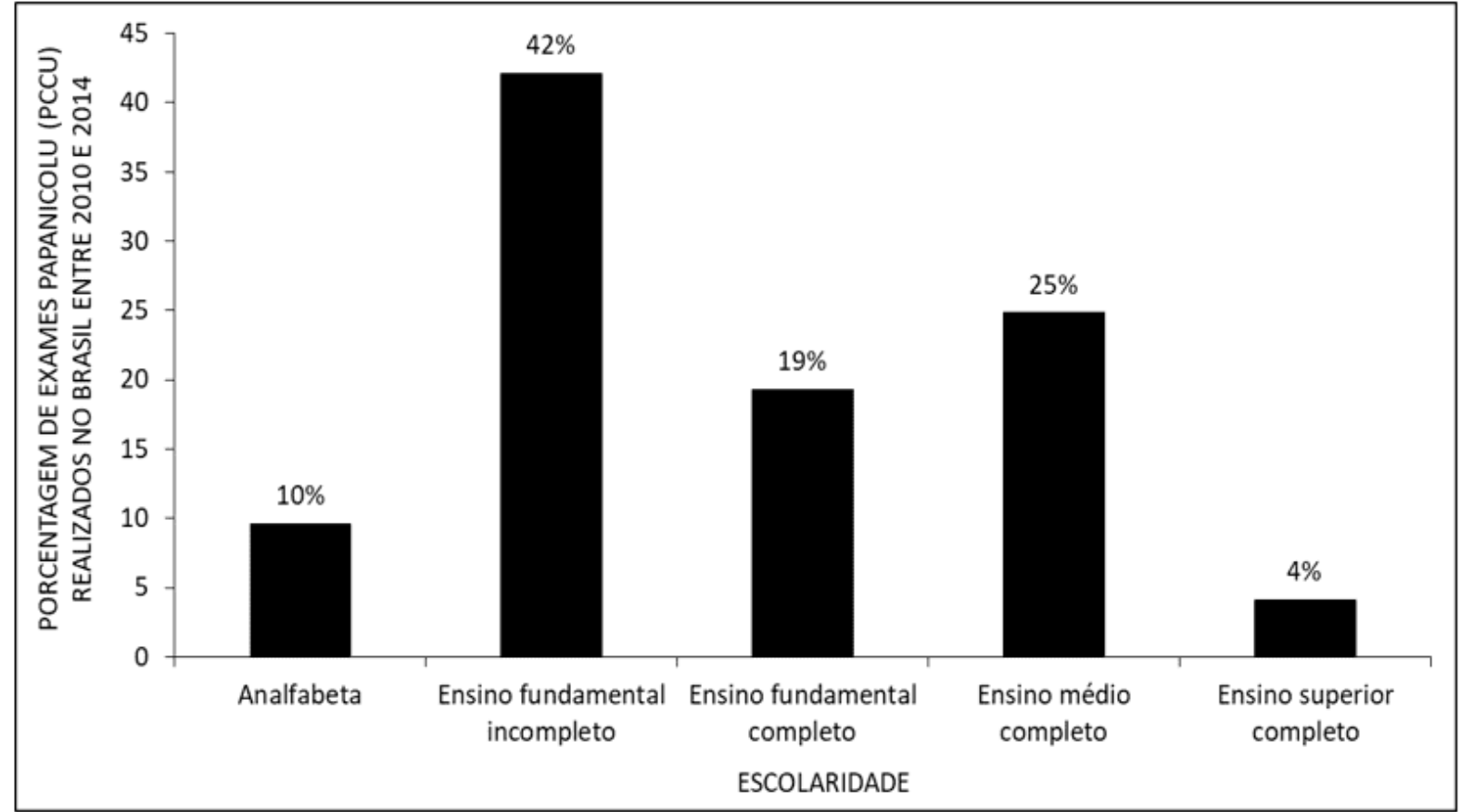

Abbildung 5 zeigt den prozentualen Anteil der Pap-Abstrich-Scans (PCCU), die in Brasilien zwischen 2010 und 2014 nach ethnischer Zugehörigkeit durchgeführt wurden. Frauen weißer und brauner Ethnischer ziehen im Vergleich zu schwarzen, gelben und indigenen Frauen eine große Anzahl von Tests durch.

RC: 67821

Disponível em:

https://www.nucleodoconhecimento.com.br/gesundheit/gebaermutterhalskrebs 
Abbildung 5 zeigt den prozentualen Anteil der Pap-Abstrich-Scans (PCCU), die in Brasilien zwischen 2010 und 2014 durchgeführt wurden, nach ethnischer Zugehörigkeit.

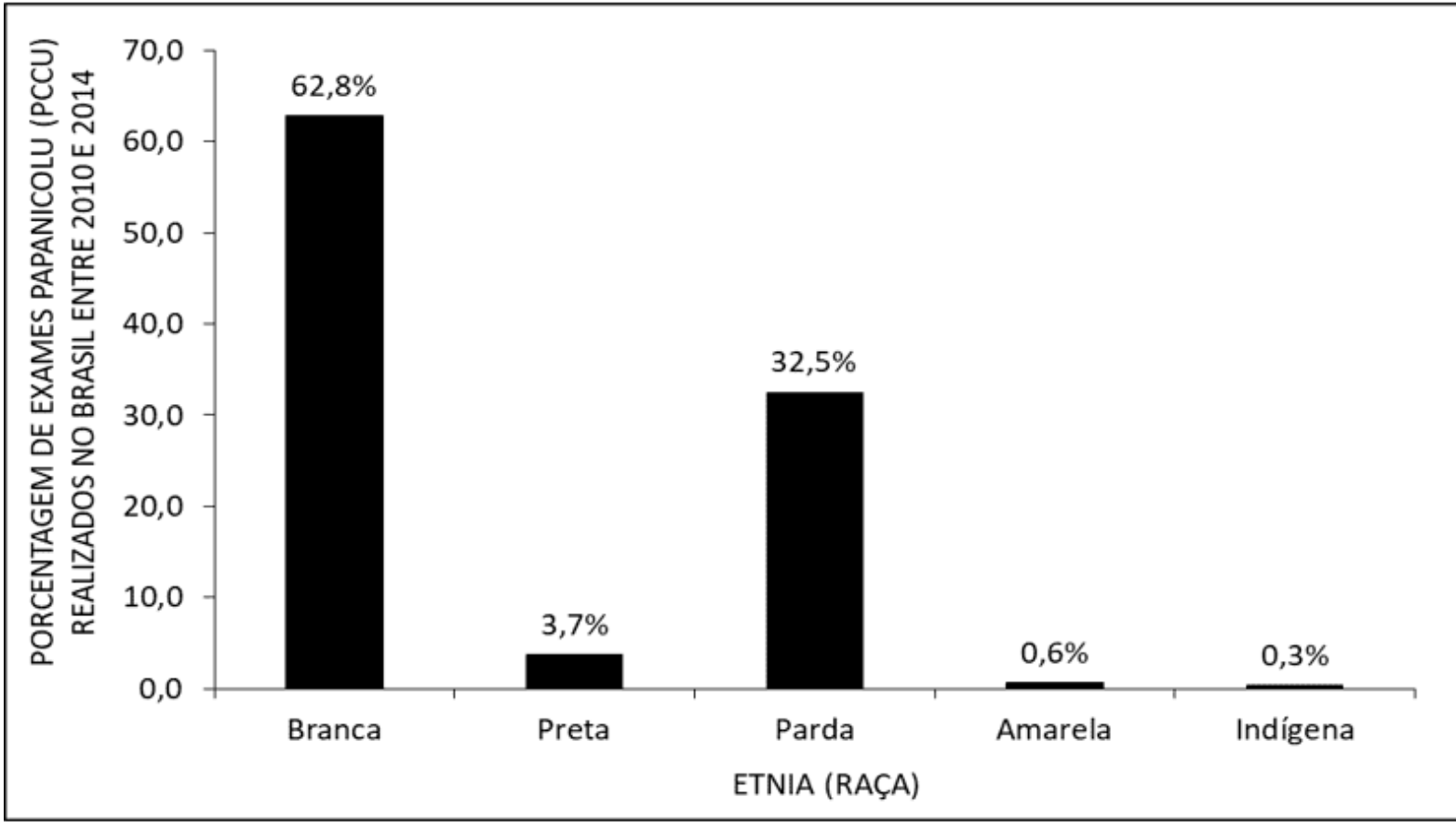

Abbildung 6 zeigt den Prozentsatz der Pap-Abstrich-Scans, die zwischen 2010 und 2014 in Brasilien im normalen Bereich durchgeführt wurden. Unter den durchgeführten Tests zeigen die Daten, dass die Mehrheit nicht im normalen Bereich liegt.

RC: 67821

Disponível em: 
Abbildung 6 zeigt den Prozentualen Anteil der Pap-Abstrichtests, die zwischen 2010 und 2014 in Brasilien im normalen Bereich durchgeführt wurden.

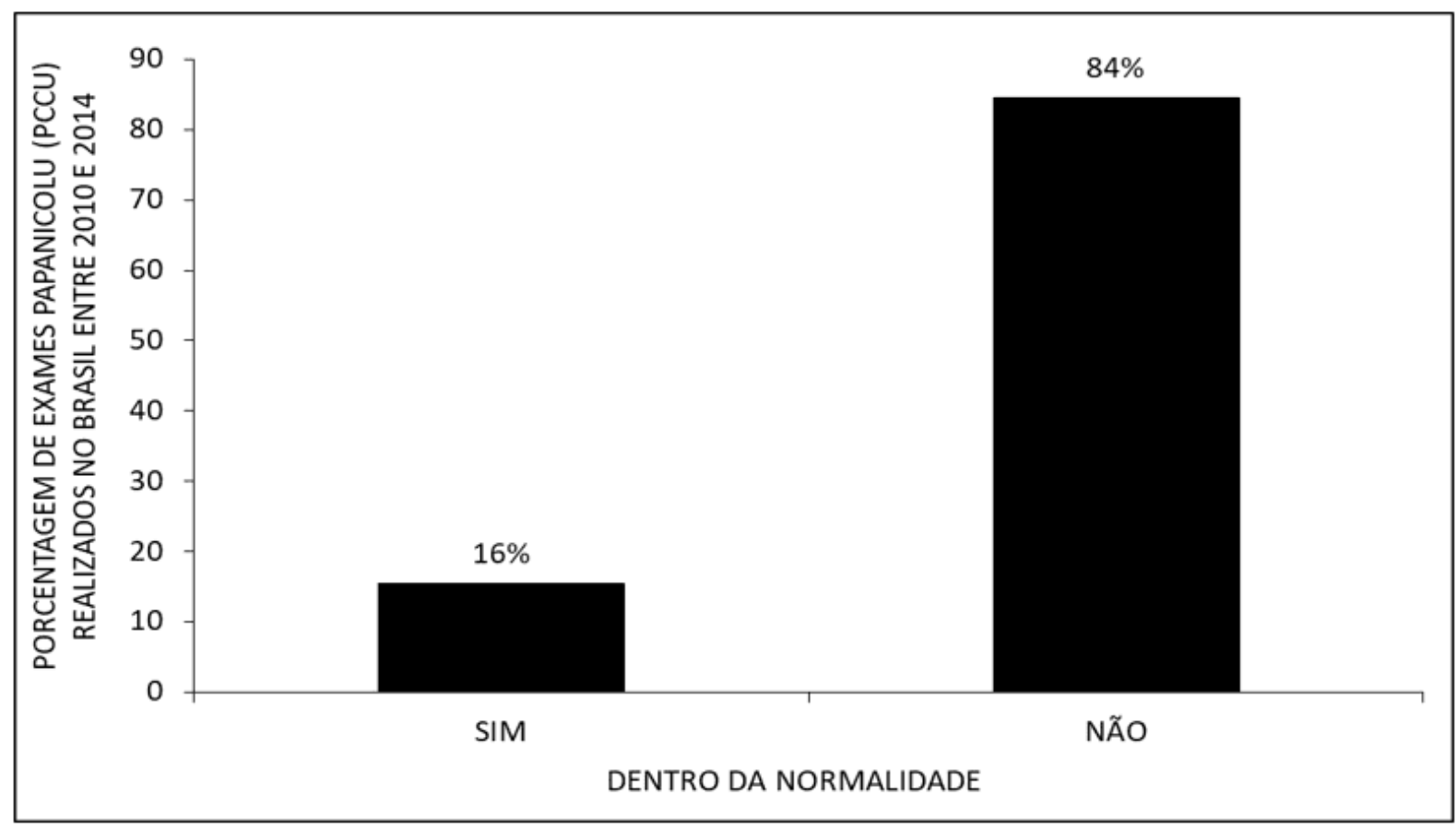

Abbildung 7 zeigt den Prozentsatz der Pap-Abstrich-Scans (PCCU), die in Brasilien zwischen 2010 und 2014 pro Sammelintervall durchgeführt wurden. Die Tests wurden meist im Intervall von bis zu 10 Tagen gesammelt, während die kleinste Menge ein Sammelintervall von mehr als 30 Tagen hat.

RC: 67821

Disponível em:

https://www.nucleodoconhecimento.com.br/gesundheit/gebaermutterhalskrebs 
Abbildung 7 zeigt den Prozentualen Anteil der Pap-Abstrichtests (PCCU), die in Brasilien zwischen 2010 und 2014 nach Sammelintervall durchgeführt wurden.

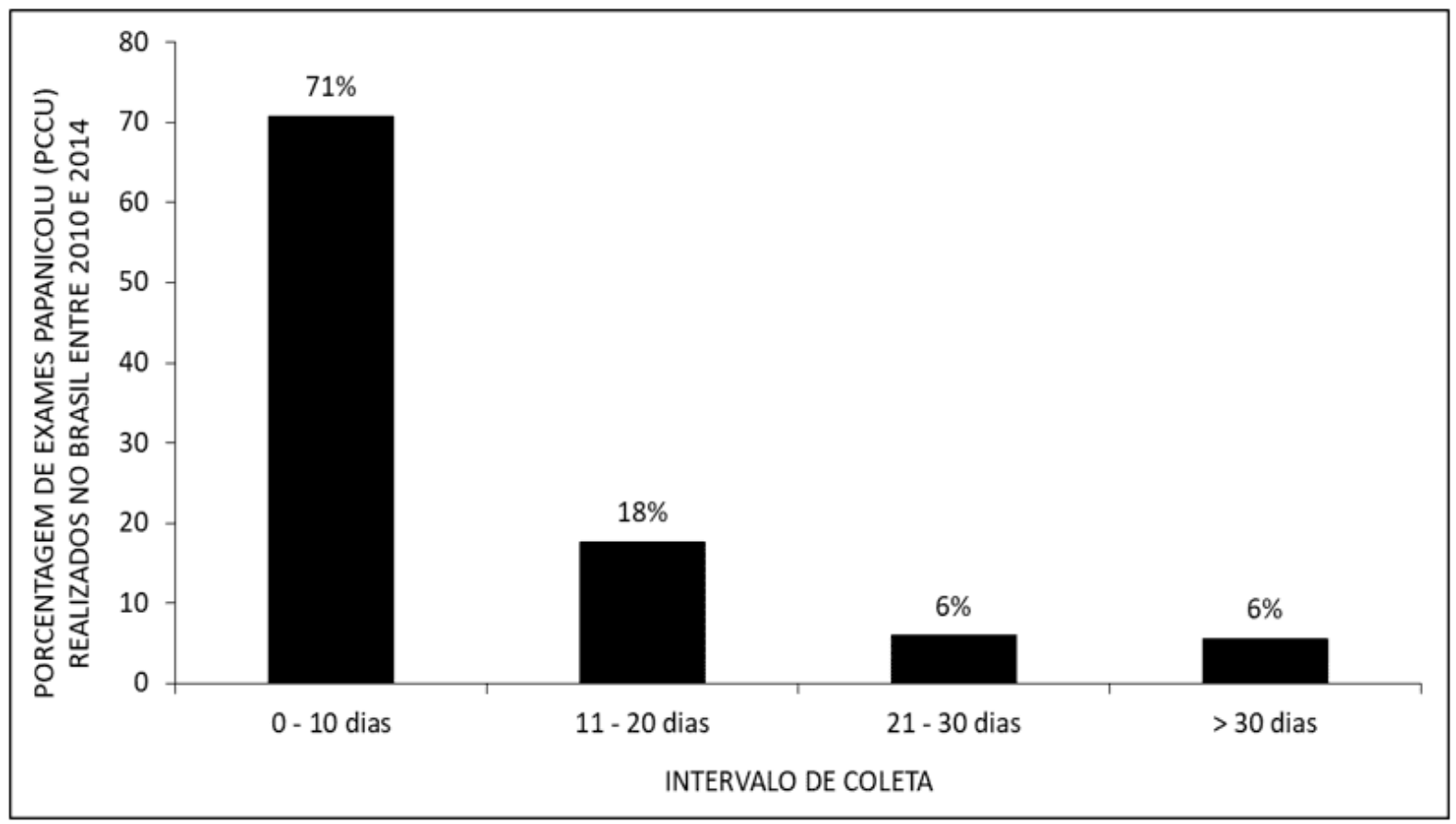

Abbildung 8 zeigt den Prozentualen Anteil der Pap-Abstrich-Scans (PCCU), die in Brasilien zwischen 2010 und 2014 durchgeführt wurden, nach Ergebnisintervallen. Zeigt an, dass die meisten Ergebnisse innerhalb von 10 Tagen veröffentlicht wurden. Während die kleinste Menge in mehr als 30 Tagen.

RC: 67821

Disponível em: 
Abbildung 8 zeigt den prozentualen Anteil der Pap-Abstrichtests (PCCU), die in Brasilien zwischen 2010 und 2014 nach Ergebnisintervall durchgeführt wurden.

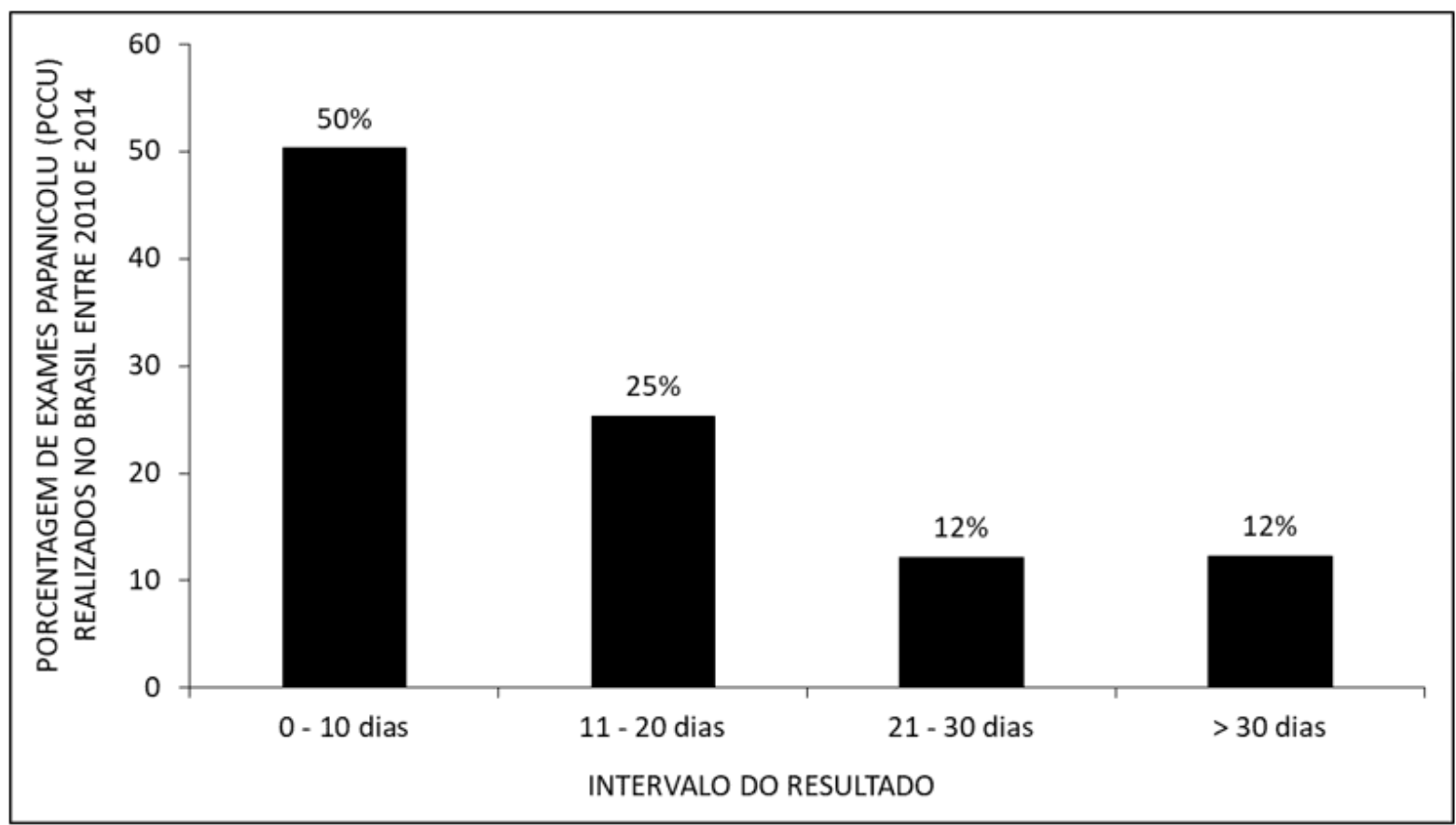

Abbildung 9 zeigt den Prozentsatz der Pap-Abstrich-Scans (PCCU), die in Brasilien zwischen 2010 und 2014 durchgeführt wurden, nach einer früheren zytologischen Untersuchung. Die meisten der befragten Frauen hatten sich zuvor Tests unterzogen.

RC: 67821

Disponível em: 
Abbildung 9 zeigt den Prozentsatz der Pap-Abstrich-Scans (PCCU), die in Brasilien zwischen 2010 und 2014 durchgeführt wurden, nach früheren zytologischen Untersuchungen.

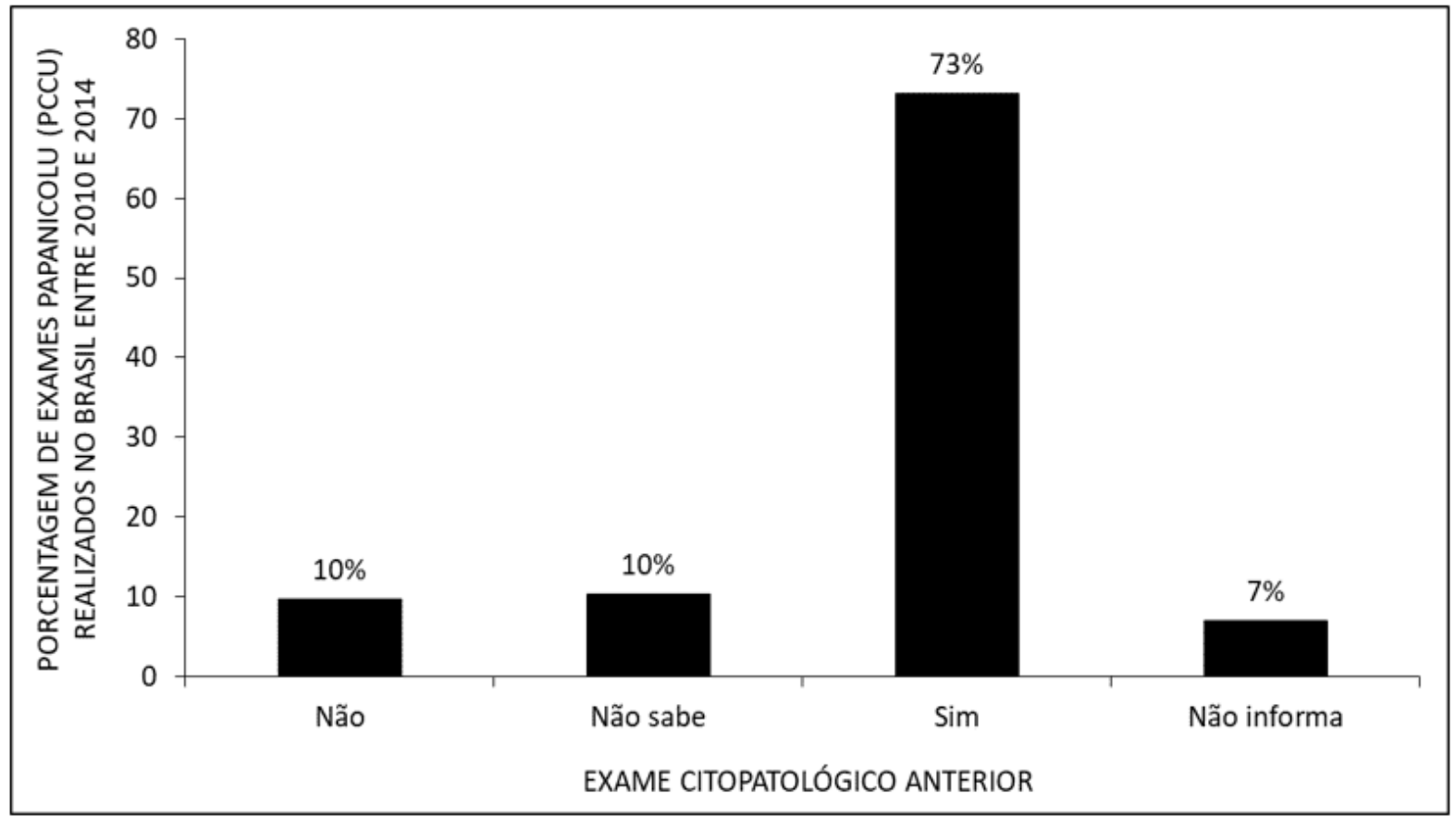

Abbildung 10 zeigt den Prozentsatz der Pap-Abstrich-Scans (PCCU), die in Brasilien zwischen 2010 und 2014 durchgeführt wurden, entsprechend dem Zeitpunkt der letzten Präventivuntersuchung. Die Daten zeigen, dass die meisten Frauen von Jahr zu Jahr Prüfungen ablegen.

RC: 67821

Disponível em: 
Abbildung 10 zeigt den Prozentsatz der Pap-Abstrichtests (PCCU), die in Brasilien zwischen 2010 und 2014 durchgeführt wurden, entsprechend dem Zeitpunkt der letzten Präventivuntersuchung.

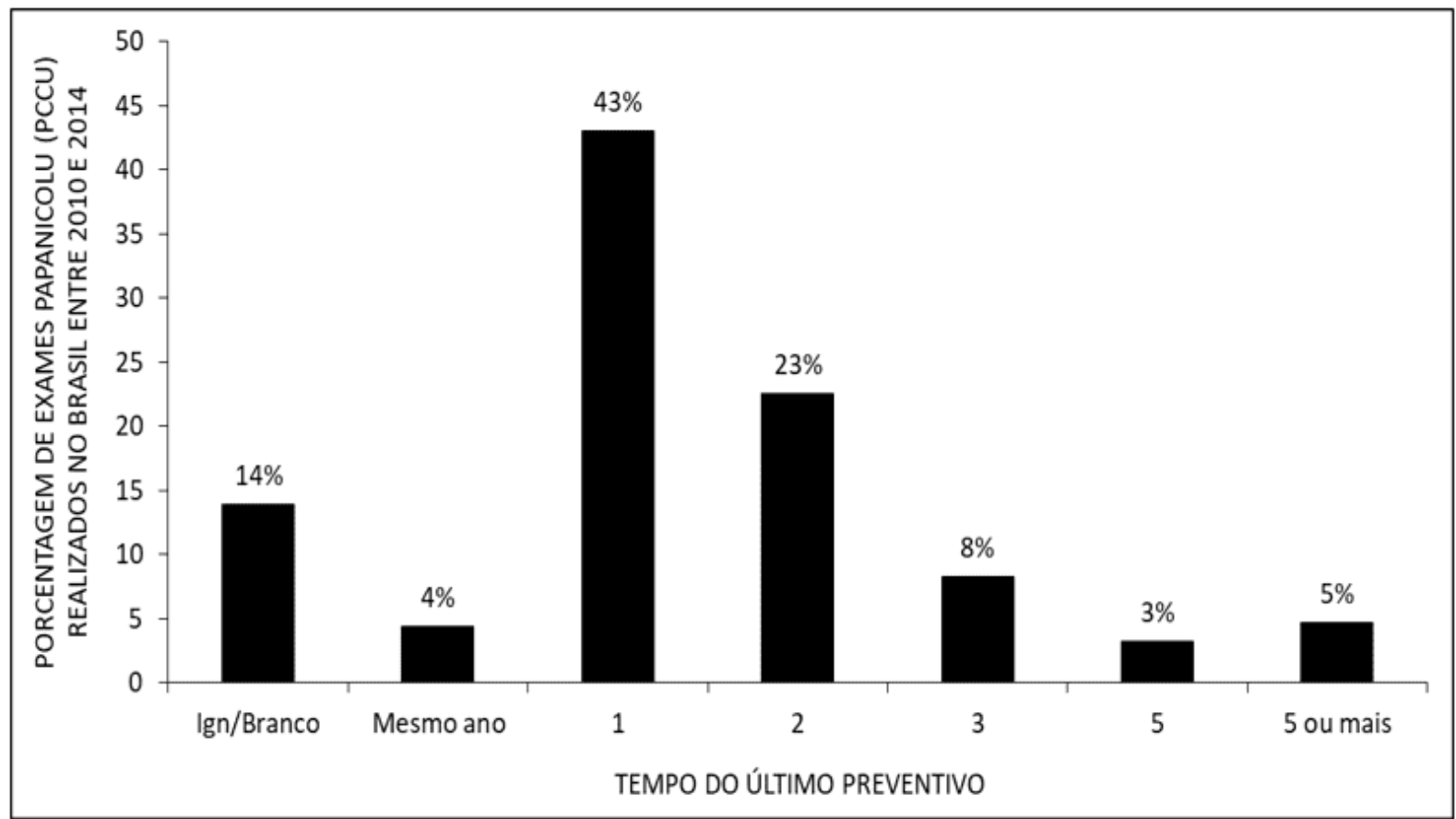

\section{DISKUSSION}

Die Daten zeigen einen deutlichen Rückgang der Leistung der Tests im Land (Abbildung 1). Einige Faktoren scheinen diesen Rückgang zu beeinflussen, wie z. B. mangelnde Kenntnis der Bedeutung der Prüfung und Angst vor der Prüfung; Scham der Exposition der Genitalorgane; Schwierigkeiten beim Zugriff auf UBS; mangelnde Vorbereitung von Fachleuten in Bezug auf den Respekt, die Beratung und die Informationen, die den Patienten über die Untersuchung zur Verfügung gestellt werden (SANTOS und VARELA, 2015).

Die Daten zeigen eine höhere Untersuchungsrate im Südosten und eine geringere Anzahl von Tests in den Regionen Nordosten, Süden, Mittlerer Westen und Norden (Abbildung 2). Dies basiert auf dem Wissen, der Information und dem Bewusstsein

RC: 67821

Disponível em: 
von Frauen bei der Behandlung von Gebärmutterhalskrebsprävention. In den anderen Regionen war die Positivität der Tests gering. Infolgedessen fand Amapa, das sich in der nördlichen Region befindet, weniger Tests, die einen Prozentsatz unter 1,0\% Positivität erreichten. Ebenso wie im Nordosten, Süden und Mittleren Westen. Es wurde festgestellt, dass diese Regionen keine Beteiligung und kein Bewusstsein für Frauen hatten, da die geringen wissenschaftlichen Erkenntnisse über die Durchführung der Prüfung ein Faktor sind, der in jedem Fall die Suche nach der pccu-Prüfung behindern kann. Einige Gründe rechtfertigen diese Tatsache, wie Scham, Gefühl der Angst, Peinlichkeit während der Untersuchung, Zeitmangel, Mangelndes Interesse und Mangel an Informationen über die zytopathologische Untersuchung (BORTOLON et al., 2012).

Die Daten zeigen, dass die höchste Anzahl von Tests bei Frauen im Alter von 30 bis 39 Jahren und die niedrigste Zahl bei Frauen bis 19 Jahren durchgeführt wird (Abbildung 3). Der Beginn der Untersuchung sollte im Alter von 25 Jahren sein, unabhängig davon, ob der Patient ein aktives Sexualleben hat oder nicht. Ein großer Teil des Pap-Abstrichs wird in den Altersgruppen von 30 bis 39 Jahren durchgeführt, so die Tabelle. Die Untersuchung sollte bis zum Alter von 64 Jahren folgen und nach zwei Tests unterbrochen werden, deren Ergebnisse negativ sind, zwei Tests sollten mit Intervallen von einem bis drei Jahren durchgeführt werden. Alle Frauen, die ein aktives Sexualleben haben oder älter als 18 Jahre sind in der Lage, die präventive Untersuchung pccu durchzuführen, aber dieser Test ist noch nicht vollständig von den brasilianischen Frauen erworben, es ist bekannt, dass Brasilien eine hohe Sterblichkeitsrate durch Gebärmutterhalskrebs hat. Dies liegt daran, dass die meisten Frauen eine Behandlung in einem fortgeschritteneren Ausmaß suchen, was am häufigsten bei Frauen im Alter von 30 bis 39 Jahren vorkommt. Nach Tabelle 3 wurde analysiert, dass die niedrigste Anzahl von Durchgeführten bei Frauen im Alter von 19 Jahren durchgeführt wird, gerade aufgrund des Mangels an Informationen über die pccu-Prüfung und der Angst, sie durchzuführen (BRASIL, 2014).

RC: 67821

Disponível em: 
Die Mehrheit der Frauen, die die Prüfung ablegen, haben eine unvollständige Grundschul- und Minderheitenhochschulbildung (Abbildung 4). In dieser Tabelle wurde festgestellt, daß je mehr Studien und Schulbildung Frauen haben, desto weniger sind sie an der Durchführung der präventiven Prüfung pccu interessiert. Der Grund für dieses Ergebnis hängt mit Überzeugungen und Einstellungen in der Gesundheit zusammen. 29,6 \% gaben an, sich nicht daran zu erinnern, warum sie nicht an dem Test teilgenommen haben. Einige Gründe sind auf Angst, Scham, Unbehagen und Schmerzen zurückzuführen. Wir haben in Tabelle 4 unerwartete Ergebnisse erzielt, weil wir eine hohe Anzahl von pccu-Prüfungen festgestellt haben, die von Frauen durchgeführt werden, die eine unvollständige Grundschule haben, weil Frauen, die keine unvollständige Schulbildung haben, im Allgemeinen dazu neigen, früher Familien zu gründen, und infolgedessen haben sie mehr Kinder als Frauen, die ein höheres Bildungsniveau haben. Folglich gehen diese Frauen häufiger zum Arzt und erhalten eine hohe Rate von Pap-Abstrich (SILVA et al.,2015).

Frauen weißer und brauner Ethnischer ziehen im Vergleich zu schwarzen, gelben und indigenen Frauen eine große Anzahl von Tests durch (Abbildung 5). Es wurde festgestellt, dass ein Großteil der Nicht-Pap-Abstriche mit ethnischer Zugehörigkeit verbunden ist, und ein Großteil dieses Widerstands hängt mit Faktoren wie Ungleichheiten von Rasse/Ethnizität sowie in Bezug auf den eingeschränkten Zugang einer bestimmten Rasse zusammen. Wie in Tabelle (5) dargestellt. Die geringe Abdeckung und mangelnde Zugänglichkeit der anderen Gruppen erklärt sich jedoch durch den Zugang und die differenzierte Behandlung, die sie haben, wenn sie am Ort der Prüfung ankommen (PINHO E JUNIOR, 2003).

Die Daten zeigen, dass die meisten der durchgeführten Tests nicht innerhalb des normalen Bereichs liegen (Abbildung 6). Ein großer Teil der weiblichen Bevölkerung ist ungewöhnlich, wenn die Pap-Abstriche durchführen. Dies liegt daran, dass Frauen versuchen, die Prüfung in einem etwas fortgeschritteneren Zustand zu machen. Dies erklärt sich auch aus der Tatsache, dass die meisten Frauen mehr als einen

RC: 67821

Disponível em: 
Sexualpartner haben, mit Tendenzen, hpV (Human Papillomavirus), eine sexuell übertragbare Krankheit, zu erkranken. Wo es eine zelluläre Desorganisation im Gewebe geben kann, aus dem der Gebärmutterhals besteht (NORONHA et al., 2005; OLIVEIRA et al., 2016)).

Die Tests wurden meist im Intervall von bis zu 10 Tagen gesammelt und die kleinste Menge hatte ein Sammelintervall von mehr als 30 Tagen (Abbildung 7). Das Erfassungsintervall ist meist kürzer und hat eine Intervalldauer von 10 Arbeitstagen. Der Fachmann liest die Klinge und eindrücke das Ergebnis während dieser Zeit, die pccu Untersuchung wird während einer gynäkologischen Konsultation gesammelt, und während der Konsultation wird ein vaginales Spekulum ohne Schmierstoffwirkung eingesetzt, was zu kleinen Beschwerden führen kann. Vor dem Sammeln sollte der Patient am Vortag keinen Sex haben. Es sollte außerhalb der Menstruationsperiode durchgeführt werden, um ein effektiveres Ergebnis zu erzielen (BRITO und NERY, 2007).

Die meisten Ergebnisse wurden innerhalb von 10 Tagen veröffentlicht und der niedrigste Betrag seit mehr als 30 Tagen (Abbildung 8). In Bezug auf den PapAbstrich unterzog sich 46,55\% der Frauen dem Test. Jedoch, 57,8\% führen in privaten Netzwerken, und die Ergebnisse der Prüfung sind viel schneller, wie die Ergebnisse der Frauen, die die pccu-Prüfung durch das SUS-Netzwerk durchführen, die eine längere Lieferzeit als erwartet hat (MURATA et al., 2012).

Die meisten der befragten Frauen hatten sich zuvor Tests unterzogen (Abbildung 9). Unter den Frauen, die die PCCU-Prüfung kennen, führen die meisten von innen die Prüfung häufig durch oder haben zuvor durchgeführt. In der Annahme, dass mit der Durchführung der pccu Untersuchung in bestimmten Zeiträumen wird vermeiden, also zukünftige Krankheiten, wie Gebärmutterhalskrebs. Da es ein effektiver Test ist, dass, wenn am Anfang diagnostiziert, es kann eine 100\% Chance der Heilung haben (BARBEIRO et al., 2009).

RC: 67821

Disponível em: 
Die Daten zeigen, dass die meisten Frauen von Jahr zu Jahr Prüfungen ablegen (Abbildung 10). Die meisten Frauen gehen zum Arzt, um GebärmutterhalskrebsUntersuchungen mit der Absicht durchzuführen, Krankheiten zu verhindern, und sie zunächst zu behandeln, um Probleme in der Zukunft zu vermeiden. Es wird empfohlen, die pccu-Prüfung jährlich von Jahr zu Jahr durchzuführen, um zu analysieren und zu bewerten, ob es eine Veränderung gab oder aufgetreten ist, wie das Ergebnis der vorherigen Prüfung war. Frauen, die sich um ihre Gesundheit kümmern, legen die Prüfung mit Zeiträumen in 1 bis 2 Jahren ab, wie abbildung (10) (PINHO und JUNIOR, 2003; MELO et al., 2019).

\section{FAZIT}

Diese Studie zeigte, dass, wenn es eine gute Leistung, um eine Krankheit als schwer zu verhindern, das Ergebnis ist zufriedenstellend und wirksam. Es ist wichtig, dass sie Enthüllungen und Informationen über die vorbeugende Untersuchung haben, aber es ist bekannt, dass die Prävention selbst nur mit der Leistung des PapAbstrichs erfolgt, um früh das Ausmaß des Grades des Krebses zu erkennen. Das, wenn auf den ersten behandelt hat eine hohe Chance auf Heilung.

Durch die Ergebnisse, die durch die Analyse der Daten zwischen den Jahren 2010 bzw. 2014 erzielt wurden, wird der Schluss gezogen, dass im Laufe der Jahre die Suche nach dem Test allmählich zugenommen hat, aber einige Aspekte blieben bestehen, wie die Scham, den pcc-Test durchzuführen, Angst, Schmerzen, unter anderem. Die Ergebnisse zeigten jedoch eine hohe Untersuchungsrate in der südöstlichen Region und einen niedrigeren Index in der nördlichen Region. Die Daten zeigen eine geringe Offenlegung und ein geringes Bewusstsein für Frauen in der nördlichen Region. Daher ist die Verbreitung, das Bewusstsein und die Durchführung der zytopathologischen Untersuchung sehr wichtig für die Prävention von Gebärmutterhalskrebs, der heute die Hauptursache für die Sterblichkeit bei brasilianischen Frauen ist.

RC: 67821

Disponível em: 


\section{REFERENZEN}

ARAÚJO, R.M.; CUNHA, H.C.O.; FERREIRA, V.G.C.; TRINDADE, M.P.; SOUZA, H.S.L. a importância da realização do exame papanicolau como instrumento de prevenção do câncer de colo do útero: uma revisão integrativa de literatura, Anais do V Congresso de Educação em Saúde da Amazônia. COESA. Universidade Federal do Pará. 2359-084. 2016.

BARBEIRO, F.M.S.; CORTEZ, E.A.; OLIVEIRA, P.A.M.C.; SILVA, A.L.O.; conhecimentos e práticas das mulheres acerca do exame papanicolau e prevenção do câncer cérvico-uterino. Cuidado é fundamental. 1(2):414-422. 2009.

BORTOLON, P.C.; SILVA, M.A.F.; CORRÊA, F.M.; DIAS, M.B.K.; KNUPP, V.M.A.; ASSIS, A.; CLARO, I.B. Avaliação da Qualidade dos Laboratórios de Citopatologia do Colo do Útero no Brasil. Revista Brasileira de Cancerologia. 58(3),435444,2012 .

BRASIL. Ministério da Saúde. Secretaria de Atenção à Saúde. Departamento de Atenção Básica. Controle dos cânceres do colo do útero e da mama / Ministério da Saúde, Secretaria de Atenção à Saúde, Departamento de Atenção Básica. - 2. ed. - Brasília: Editora do Ministério da Saúde, 2014. 124 p.

BRASIL. Ministério da Saúde. Secretaria de Atenção à Saúde. Departamento de Atenção Básica. Controle dos cânceres do colo do útero e da mama. Ministério da Saúde. 2. ed. - Brasília: Editora do Ministério da Saúde. 124 p. 2013.

MELO, Carolina Simas. Et al. Caracterização epidemiológica dos óbitos no Brasil por macrorregião de 2016 a 2018. Revista Científica Multidisciplinar Núcleo do Conhecimento. Ano 04, Ed. 12, Vol. 01, pp. 05-17, 2019. Link de acesso: https://www.nucleodoconhecimento.com.br/saude/obitos-no-brasil

RC: 67821

Disponível em: 
MENETRIER, J.V.; BOING, A.; MEDEIROS, K.A. Alterações citopatológicas do colo uterino em mulheres atendidas na 8a Regional de Saúde do Paraná no ano de 2014. Espaço para a Saúde - Revista de Saúde Pública do Paraná. V. 17, n. 2, p. 169177, 2016.

MURATA, I.M.H.; GRABRIELLONI, M.C.; SCHIRMER J. Cobertura do Papanicolau em mulheres de 25 a 59 anos de Maringá - PR, Brasil. Rev Bras Cancerol.58(3):409-15, 2012.

NORONHA, V.L.; NORONHA, R.; CARMONA, B.; MACEDO, L.A.; CRUZ, E.M.; NAUM, C.; MELLO, W.; VILLA, L. Papilomavírus humano (hpv) em mulheres com citologia oncótica dentro dos limites da normalidade. ARTICLE. 17(1): 49-55, 2005.

OLIVEIRA, R.C.E.. et. al. Perfil epidemiológico do HPV da população feminina sexualmente ativa, na faixa etária de 10 a 80 anos. Revista Científica Multidisciplinar Núcleo do Conhecimento. Ano 01, Ed. 07, Vol. 04, pp. 21-34, 2016. Link de acesso: https://www.nucleodoconhecimento.com.br/saude/hpv

PINHO, A.A; JUNIOR, I.F. Prevenção do câncer de colo do útero: um modelo teórico para analisar o acesso e a utilização do teste de Papanicolaou. Rev. Bras. Saude Mater. Infant. n.1 Recife jan./mar. 2003.

RICO, A.M.; SILVA, G.A.; LOWY, I.; TEIXEIRA, L. (Org.) et al. Câncer de mama, câncer de colo de útero: conhecimentos, políticas e práticas. Outras Letras. 256 p. 2015.

SANTOS, A.C.S.; VARELA, C.D.S. Prevenção do câncer de colo uterino: motivos que influenciam a não realização do exame de papanicolaou. Revista Enfermagem Contemporânea. 4(2):179-188, 2015.

$\mathrm{RC}: 67821$

Disponível em: 
SILVA, M.A; TEIXEIRA, E.M.B; FERRARI, R.A.P; CESTARI, M.E.W; CARDELLI, A.M. Fatores relacionados a não adesão à realização do exame de Papanicolau. Rev Rene. 16(4).532-9. 2015.

Eingereicht: November 2020.

Genehmigt: November 2020.

RC: 67821

Disponível em:

https://www.nucleodoconhecimento.com.br/gesundheit/gebaermutterhalskrebs 